\title{
A Social Network of Collaborating Industrial Assets
}

\author{
Hao Li ${ }^{1}$, Adrià Salvador Palau and Ajith Kumar Parlikad
}

\begin{abstract}
The loT (Internet of Things) concept is being widely regarded as the fundamental tool of the next industrial revolution Industry 4.0. As the value of data generated in social networks has been increasingly recognised, Social Media and the Internet of Things have been integrated in areas such as product-design, traffic routing, etc.. However, the potential of this integration in improving system-level performance in industrial environments has rarely been explored. This paper discusses the feasibility of improving system-level performance in industrial systems by integrating Social Networks into the loT concept. We propose the concept of a Social Internet of Industrial Assets (SloIA) which enables the collaboration between assets by sharing status data. We also identify the building blocks of SlolA and characteristics of one of its important components - Social Assets. A sketch of the general architecture needed to enable a Social Network of Collaborating Industrial Assets is proposed and two illustrative application examples are given.
\end{abstract}

\section{Keywords}

Internet of Things, Social Networks, asset management, diagnostics and prognostics, condition-based maintenance, collaborating decision-making

\section{Introduction}

The large amounts of data generated by well-instrumented assets, together with the rapid development of Information Communication Technologies, has led to growing applications of the IoT concept in industry. During the past years, quite a few IoT applications have been seen in various aspects of the current industrial practices including environmental monitoring, inventory and production management, food supply chains (FSC), transportation, security, and surveillance (Li et al. 2012).

As an important aspect of industrial management, effective asset management is key to reducing the total cost of asset ownership while improving machine availability, guaranteeing security, and increasing productivity. In recent years, the IoT has been increasingly regarded as an effective framework to improve asset management policies, allowing asset managers to have a much broader knowledge of their asset fleet (Zhang et al. 2014), (Lee et al. 2015). As a result of this, the notion of SIoT (Social Internet of Things), which results from integrating Social Media into the IoT, has been implemented in application areas such as product lifecycle management (Cai et al. 2014), traffic routing (Schurgot et al. 2012), and workplace help and support (Kranz et al. 2010).

Although quite a few circumstances exist where enhancing social behaviour of industrial assets are likely to be beneficial, the potential of this integration in improving system-level performance in asset fleets has rarely been explored. For instance, in a Social Network, a fleet of assets with similar characteristics could share their diagnostics and prognostics knowledge gained by learning from their own condition data. This could help assets to improve their prognostics accuracy, and also to identify latent problems which would be difficult to notice with only the information available to an asset itself.
Following the SIoT concept, this work attempts to explore the possibility of improving asset management performance by developing a Social Network of Collaborating Industrial Assets for knowledge and data sharing between machines.

Section 2 reviews recent developments in SIoT, use of distributed decision-making systems in different aspects of maintenance optimisation, architectures and frameworks proposed for IoT and SIoT, as well as evolution of what we call Smart Objects. In section 3, our vision of Social Assets are presented and the fundamental properties needed to transform Smart Assets into Social Assets are discussed. Section 4 outlines the building blocks for a Social Internet of Industrial Assets (SIoIA), and presented a general architecture for SIoIA. Subsequently, two illustrative examples of SIoIA applications are presented in section 5. Section 6 provides a guideline on future work. Conclusion of the paper is given in section 7 .

\section{Literature review}

The term IoT was first coined in 1999 by The Auto-ID Labs, within the context of supply chain management enabled by RFID (radio-frequency identifications) technology (Gubbi et al. 2013). However, its current definition has been extended to include a dynamic global network infrastructure with self-configuring capabilities, where physical and virtual things have identities, physical attributes, and virtual

\footnotetext{
${ }^{1}$ Department of Engineering, Institute for Manufacturing, University of Cambridge, Cambridge, CB3 OFS, UK

Corresponding author:

Hao Li, Department of Engineering, Institute for Manufacturing, University of Cambridge, Cambridge, CB3 OFS, UK

Email: h1433@cam.ac.uk
} 
personalities, use intelligent interfaces, and are seamlessly integrated into the information network (Van Kranenburg 2008). Today, vast amounts of data are generated and shared across the IoT (Zaslavsky et al. 2013). Examples range from self-driving cars (Gerla et al. 2014) and continuously monitored gas turbines to the immensely popular smart phones, GPS enabled wristbands and other wearables.

Smart phones differ from other IoT-powered devises in that they are designed, operated and marketed as a prominently social tool. Profiting from this social dimension, the data generated by such phones has been widely used in the consumer world to benchmark and optimise product quality and customer experience. For instance, companies like Garmin, Nike and Microsoft have provided platforms for consumers to share and compare exercise data collected via smart phones and other smart gadgets (De Saulles 2016).

Besides smart phones, the notion of incorporating social elements to the IoT has been around for approximately a decade, leading to the development of SIoT. One of the early ideas associated with SIoT is "Blogject", a neologism meaning "things that blog". An example was a flock of pigeons that were equipped with telematics for wireless communication, a GPS device for track tracing, and sensors to record the content of air pollutants (Bleecker 2006) . The potential of combining social and technical networks has also been tested on service provision to both human users and technical systems. A use-case of a socio-technical network - The Cognitive Office, was reported, where Twitter was used to enable an online social network for objects in a smart office to post events from selected sensors and listen for Tweets from other devices (Kranz et al. 2010). The exploitation of SIoT can also be found in traffic routing problems, such as opportunistic communication enabled by social networks in dynamic traffic networks (Schurgot et al. 2012). Extending beyond objects socialising with each other, the integration of humans into SIoT has also been discussed, adding the human-to-thing element to achieve the complete vision of SIoT (Ortiz et al. 2014).

The growing volumes of available data generated by modern industrial assets equipped with sensors, and the concurrent development of advanced data analytic tools and artificial intelligence has naturally led to discussions of the SIoT in the context of industrial assets. An inceptive application of the SIoT focuses on trying to improve industrial system performance by making use of distributed decision-making with the help of the IoT, giving a social dimension to industrial assets. For example, the attempts of using distributed decision-making in production scheduling, maintenance scheduling, and inventory management can be found in (Lim et al. 2009), (Zhou et al. 2004), and (Jiang and Sheng 2009) respectively.

In some cases, the social element in these approaches is not the human operator, like in the case of smart phones, but the asset itself. In other cases both humans and machines act socially. In this paper, we focus in the cases where most of the social collaboration is performed by industrial assets, and where human agents are limited to setting the system constraints. In order to set up such systems, one must first address the understanding of distributed artificial intelligence techniques.
The introduction of distributed artificial intelligence, mainly based on agent-based systems and holonic manufacturing systems paradigms, is usually aimed to satisfy production requirements such as customisation, agility, flexibility, and robustness. A common distributed artificial intelligence approach is to use Multi-Agent Systems (MAS), which can be defined as "distributed systems of independent actors, called agents, that cooperate or compete to achieve a certain objective" (Tuyls and Weiss 2012). Broadly, agentbased systems have been designed for one of the following purposes: maintenance resource integration, machinery fault diagnostics and prognostics, and maintenance scheduling. The rest of the section surveys recent advance of agent-based systems in the two latter areas which are of more relevance to the purpose of this paper.

A Multi-Agent System-based reference model for fault management system (FMS) has been developed by Cerrada et al. (2007). The FMS can be integrated with the supervision applications to support the decision-making on the controlled processes including component monitoring, failure detection, failure prediction, maintenance scheduling, and maintenance plan execution. A prototype of the reference model has been implemented on Java Development Framework (JADE) to a pool pumping system as a case study. Cerrada's paper had followed from a line or research starting in the early 2000's: arguing that distributed fault detection and handling is a more suited paradigm for fault management systems, Ouelhadj et al. (2000) described a multi-agent architecture for distributed and realtime monitoring. The major functions of the monitoring system are performed via the information exchange and co-ordination based on Contract Net Protocol (CNP) between a set of Resource Monitoring Agents (RMA) each responsible for a manufacturing resource. Focusing on data interpretation and condition monitoring applications, Mangina et al. (2000) introduced a hierarchical decentralised multi-agent architecture named COMMAS. Unlike the work of Ouelhadj et al. (2000) where one agent is assigned to one production resource to perform a wide range of information finding tasks, the agents in COMMAS each represent one aspect of application so as to distribute the responsibilities of information processing. Three hierarchical categories of agents responsible for data fusion, cross sensor corroboration, and reasoning and decision-support functions, respectively, have been proposed. In a later work (McArthur et al. 2004), the proposed COMMAS architecture was implemented for the design of a multiagent transformer condition monitoring system using $\mathrm{K}$ means clustering, rule induction, and a back-propagation neural network. Another work of his (McArthur et al. 2005) developed an anomaly detection system employing an extended COMMAS architecture. An infotronics-based prognostic prognostics tool called The Watchdog Agent was proposed for product performance degradation assessment and prediction (Djurdjanovic et al. 2003). The Watchdog Agent is capable of diagnosing the current state and prognosticating the future state of its objective component based on the readings from multiple sensors.

The multi-agent paradigm, commonly used in reactive and dynamic production scheduling, can also be adopted for maintenance scheduling problems. Coudert et al. 
(2002) proposed a production and maintenance integrated scheduling system called the RAMSES-II (Reactive Multiagent System for Scheduling) based on fuzzy logic. In this framework, every manufacturing resource is associated with a machine agent in charge of bidding for production tasks, a maintenance agent that creates maintenance task orders, and a negotiation agent that reconciles the conflicts between the machine and the maintenance agents to get the maximal aggregated degree of satisfaction of two parties. Blackboards are employed to provide a virtual Gantt chart view of the ongoing negotiation process at different conceptual levels. Targeting at the same problem in a flow shop, Khelifati and Benbouzid-Sitayeb (2013) proposed a similar agent-based approach comprised of machine agents and maintenance agents to simultaneously schedule production and periodic maintenance. A bus maintenance scheduling method based on multi-agent systems was proposed by (Zhou et al. 2004) to make up for the downsides of centralised scheduling while dealing with unforeseen events. The model is formulated using three layers and four types of agents to heuristically schedule incoming maintenance tasks cooperatively using CNP. An agent-based system for dynamic scheduling of maintenance tasks in the petroleum industry using reinforcement learning was developed by Aissani et al. (2009), and put into experimentation to an Algerian petroleum refinery. The system consists of 'resource agents' for the pumps, 'parts agents' for the tanks containing oil, and an 'observer agent' that has a global view of the system. Their work differs from the aforementioned researches in that there is no centralised decision-making mechanism since each resource and part agent decides its next action depending on its own knowledge base. Feng et al. (2012) employed MAS with a two-layer structure for online CBM decision-making among a missionoriented aircraft fleet considering the constraint of limited maintenance resources. The coordination takes place at two levels both following a heuristic rule-based negotiation mechanism: the local scheduling decision is made via negotiation between Aircraft agents and Maintenance Agents representing maintenance teams while the global scheduling is done by the Management and Coordination Agent. Also dealing with aircraft fleets, Brintrup et al. (2011), collaborated with Boeing in order to enable its assets to become "Self-Serving Assets", with the goal "for assets to autonomously plan their own service and maintenance while collaborating with service and maintenance providers and other assets".

As the underlying technologies of the IoT concept have taken shape, research efforts towards the integration of these various technologies have started to produce architectures and frameworks for the IoT and SIoT. Sánchez López et al. (2012) designed an IoT architecture within a Smart Object framework. The proposed architecture components include Smart Objects, network protocols, interfaces, and events and repository databases. A prototype of the architecture for the real-time monitoring of goods in supply chains was implemented using Wireless Sensor Networks and Web Services to show its feasibility and flexibility. Zhang et al. (2014) extended the techniques of the IoT to the manufacturing field and developed a four-layer architecture mainly for real-time information capturing and dynamic monitoring and controlling for the manufacturing execution stage. Similarly aimed at industrial environment, Ungurean et al. (2014) presented an IoT architecture composed of a data server module and a client appplication module based on OPC.NET specifications. A five-level Cyber-Physical Systems structure has been proposed for Industry 4.0 manufacturing systems (Lee et al. 2015). The corresponding algorithms and technologies at each system layer have been suggested for the desired functionalities of the overall system. Guo et al. (2013) has proposed a reference architecture for opportunistic IoT which exploits the potential benefits of human social behaviour in the IoT. Focusing on a specific instance of SIoT, an architecture based on vehicular ad-hoc networks has been proposed that identifies social structures and related interactions of vehicles in the machine-to-machine social networks (Alam et al. 2015). An SIoT architecture following the three-layer model made of the sensing, network, and application layers has been presented by Atzori et al. (2012), where the Social components belong to the application layer.

The existing IoT and SIoT architectures and frameworks all have the same constituent element - what is called 'Smart Object' or 'Intelligent Object'. The very first architectures of the IoT are based on the success of RFID technology. While this approach is ideal in tracking physical objects within a confined space, it is insufficient in complex situations as the objects themselves have no analytic or decision-making capabilities. This has led to enhanced requirements for objects to be smart. For instance, in the work of (Kosmatos et al. 2011), RFID-tagged objects are integrated with its online abstraction positioned with application logic. Arguing that the characteristics of software agents are very similar to those of smart objects, Fortino et al. (2012) propose a multi-layered agent-based architecture for the development of Smart Objects. Kortuem et al. (2010) categorised Smart Objects in to three levels according to their degree of awareness, representation, and interaction.

As shown in this literature review, "Smart Objects" have been around for some time, and its connection to Asset Management has been proposed before by, for example, Brintrup et al. (2011) associated to the idea of SelfServing Assets. However, this connection has only recently been made, and specifics for how to enable inter-asset collaboration are missing in literature. In this paper, we address the problem of inter-asset collaboration in asset fleets, giving specific examples and detailing the properties of the building blocks of such a Social Network of Collaborating Industrial Assets.

\section{Social assets}

Smart Objects can be described as autonomous physical/digital objects augmented with sensing, processing, and network capabilities (Kortuem et al. 2010). What it takes for objects to be 'smart' still applies to the basic components for a Social Network of Collaborating Industrial Assets in the manufacturing domain (i.e., machines or assets). However, as modern production process is often complex and requires co-efforts from a fleet of various assets, instead of just a single asset, interactions and mutual understanding between assets inevitably play a vital role. For instance, in a quarrying 


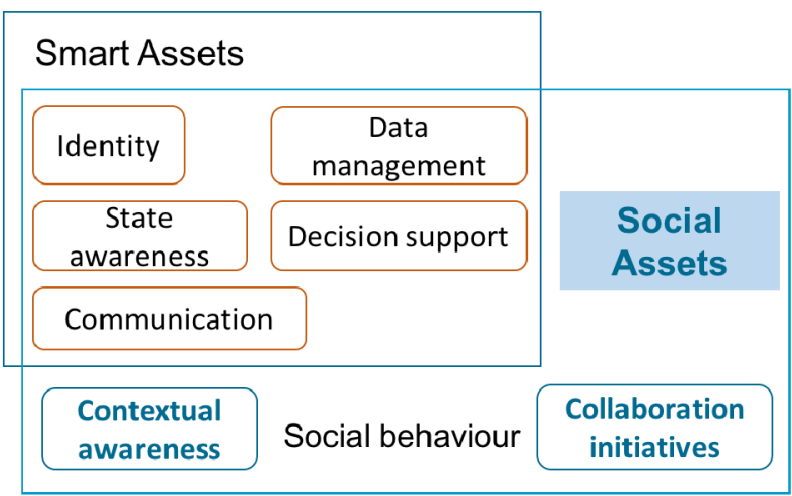

Figure 1. Fundamentals for Smart Assets and Social Assets

process where rocks are crushed and extracted from the mountains and transported by loaders to a conveyor belt, a loader without social capabilities will continue loading rocks to a conveyor belt close to failure, this may lead to severe disruptions if there is a lack of maintenance resource when the failure happens. Thus, apart from being 'smart', these components also need to be 'social'.

In order to characterise Social Assets, it is useful to first review the properties assets are supposed to possess to be 'smart'. This corresponds to the five characteristics of Smart Products/Objects proposed in the new generation of objectcentric industrial paradigm (Sánchez López et al. 2012):

Identity - A unique identity is needed for the Smart Asset to be tracked, recorded, and referred to in the system.

State awareness - Smart Assets should be able to sense its own operating status and measure performance indexes, such as vibration, temperature, and concentration of a particular chemical element, etc..

Communication - They should be capable of exchanging information with other Smart Assets, OEMs, and centralised servers.

Data management - It is necessary to classify and store measurements made by sensor transducers associated with the assets, while removing some history data, if appropriate. Decision support - Smart Assets should have a decision support mechanism to generate apt actions in response to different situations that have arisen. Moreover, they should be able to exhibit opportunistic, goal-directed behaviour and act proactively.

One of the earlier works to systematically discuss the characteristics of Social Objects and policies needed to establish a navigable SIoT is done by Atzori et al. (2012). In a later work, Atzori et al. (2014) discussed the main features that a SIoT will possess once the aforementioned basic relationships have been established. However, the fundamental properties needed for objects to socialise and collaborate, in particular in industrial environment, are not sufficiently explored.

In our vision, in order for machines to cooperate, as a means to optimising system-level performance, two more fundamental properties are needed - contextual awareness and collaboration initiatives. This transforms Smart Assets into Social Assets, as shown in Fig. 1.

Contextual awareness - A piece of asset with contextual awareness not only investigates its own status, but also perceives its environment, which may be the physical world, a user, a collection of other assets, or the Internet (Fortino et al. 2012). Assets should have an understanding of the consequence of any action they take that might cause changes to the surroundings, and also the impacts that the behaviour of other participants in the Social Network would have on themselves. Contextual perception implies understanding of a problem domain much broader than a single asset could than the boundaries of the asset itself, thus enabling the asset to act more wisely. Being contextual aware differs from being only connected in that with the same information obtained from the network, the asset not only perceives the information as it is, but also incorporates it in a broader context to better understand the complex interactions that can be triggered by this piece of information. This is also one of the basic prerequisites to plant collaboration initiatives in assets. As in the example of the loader and conveyor mentioned earlier, if the loader and conveyor are only connected with each other with no contextual awareness, the loader would still understand that the conveyor is failing. However, the loader will not be able to figure out what action to perform to lower the degradation rate of the conveyor. Whereas with contextual awareness, the loader would easily figure out that a better idea would be to reduce the amount of rocks to be loaded on the conveyor belt, which will keep the quarry operating until maintenance resource is in place.

Collaboration initiatives - Collaboration initiatives here refers to the fact that an asset views itself as closely related to other assets and is constantly seeking the opportunity to collaborate with other machines to achieve a systemlevel goal. Enabling assets to have collaboration initiatives is considered necessary in manufacturing systems for two reasons. First, the local goals of a single asset are very likely to be contradictory to the goal of a plant manager or another asset (e.g., two machines push production tasks to each other so as to avoid deterioration and wear and tear while the plant manager wants maximum productivity). If assets are open for cooperation, these conflicts can be resolved through exchange of knowledge, negotiation between machines, and collective decision making. In the example given, the machine with less severe degradation for now could offer to take on more load in exchange for less workload when later its degradation level has risen. Second, just as humans help and give advice to each other, through cooperation initiatives, assets will be inclined to recommend assets of similar status, based on their own experience, the best possible action path to take for the asset itself, as well as for the overall improvement of system-level performance.

\section{The building blocks for Social Network of Industrial Assets}

While Social Assets act as the main building block, other components should also be added during the actual implementation of SIoIA. The building components of SIoIA and their function blocks are shown in Fig. 2. A detailed description is given as follows:

Social Assets - A software agent is assigned to each asset, augmenting it with intelligent and social behaviours. These assets should be actively engaged throughout the production process. Specifically, Social Assets should be able to carry 
out:

(1) Self-monitoring, diagnostics, and prognostics: Social Assets should be equipped with algorithms to effectively process status data obtained by sensors and events data. These algorithms should be able to store the analysis outcome in an easily accessible way. There are two potential benefits of doing so: a) providing a model for near real-time machine status evaluation, fault diagnostics, and remaining useful life prediction; b) allowing fast improvement of the model when new information channel is available. In particular, for the case of a network of Social Assets, new information channels can be built instantly since all machines are actively engaged in communication with others.

(2) Relationship analysis: just like human beings interacting with each other in different ways on the basis of relationships, Social Assets also act differently when faced with other Social Assets based on dynamic metrics quantifying their closeness and similarity. The ability to perform relationship analysis is necessary in certain situations if improved asset performance is to be achieved. For instance, machines of the same type and configuration operating under similar conditions can be assumed to have similar degradation behaviour and thus they can choose to share status data with each other to build more accurate diagnostics and prognostics models of their own. One approach of defining similarity can be via a set of inter-asset similarity metrics with the element in row $i$ and column $j$ defining the soundness of sharing the data of asset $j$ with asset $i$. This approach will be further elaborated in next section.

(3) Selection of data of interest: with the large amount of data generated by a Social Network of Assets comes the trade-off between the cost of heavy computation, and the cost of myopic decisions resulted from using incomprehensive information. For a single Social Asset, the selection of data of interest needs to be executed in two directions and with reference to its relationship analysis outcome: a) the type of data to post on the Social Network; b) the subscription to certain data that may be of self-interest.

(4) Collaboration functions: various forms of collaboration opportunities exist between Social Assets, of which data sharing is just one starting point. With a reasonable understanding of other elements in its surroundings, a Social Asset can give advice to a similar asset based on its own experience, offer to share the load of another asset where necessary, act as a media for connecting other assets of potential mutual interest, etc.

Social Network Platform - A social platform is where the actual social behaviours of assets occur. It should be equipped with necessary hardware and software to function as a channel for:

(1) General communication: it should enable a) machines to post production-related data, b) machines to subscribe to their own information of interest, and c) M2M (machine-tomachine) communication.

(2) Resource administration: acting like a yellow page to manage assets registration data and provide a directory service.

(3) Data storage and management: efficiently storing and deleting data shared on the platform.

Research work dedicated to the development of SIoT and Io $\mathrm{T}$ architectures that can be extended to meet these demands

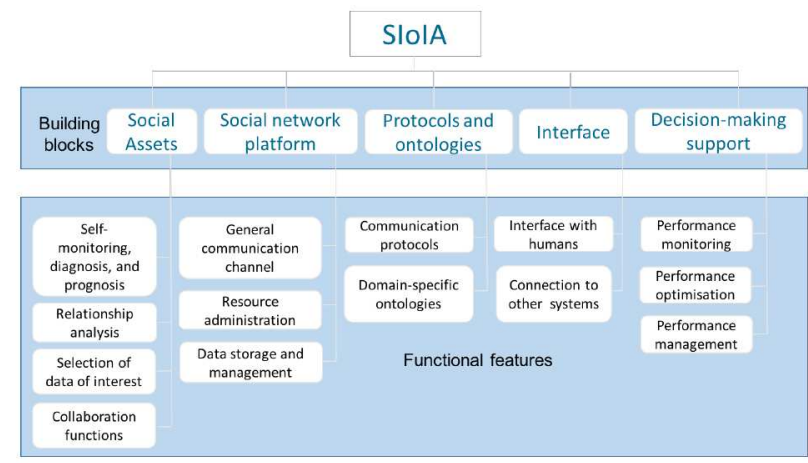

Figure 2. SlolA building blocks and their functional features

can be found in Atzori et al. (2012); Ungurean et al. (2014); Sánchez López et al. (2012).

Protocols and ontologies- Protocols are needed to guarantee a unified data transport format while ontologies provide a mutually understandable communication context. A detailed review of existing protocols in support of the IoT is given in Al-Fuqaha et al. (2015). Examples of ontologies that can be extended or modified to suit this purpose can be found in Lemaignan et al. (2006); Geerts and O'Leary (2014).

Interface- An interface is needed for data sharing with other systems as well as human operators:

(1) interaction with humans: although ideally human interventions with the system would be minimal, an interface is needed for human-computer interaction such as receiving parameter inputs, getting commands, sending alerts, and suggesting actions;

(2) connection to other systems: channels for information exchange with other systems regarding service, finance, and logistics are needed for decision making at the organisation level.

Similar requirements for interfaces have also been mentioned in Iung et al. (2009).

Decision-making support- A series of decision-making support functions as listed below should be available. However, it still remains to be explored whether the function should reside in individual assets, at a supervisory level, in a hybrid combining these two, or through other manners.

(1) performance monitoring: Production data sent to the social platform is used by the performance monitor to assess individual asset performance and system performance. The performance monitor can serve to, for instance, identify the bottleneck of the manufacturing plant. At a higher level, by connecting with other relevant databases of the organisation, potential risks to the organisation's objectives can be identified;

(2) performance optimisation: The purpose of the performance optimiser is to generate optimal action plans based on knowledge provided by the performance monitor. The consequent system management plans are then passed on to the performance manager for further consideration;

(3) performance management: The major function of performance management is to select the best system management plan proposed by the performance optimiser and post it on the Social Network Platform to pass to relevant assets to implement. 


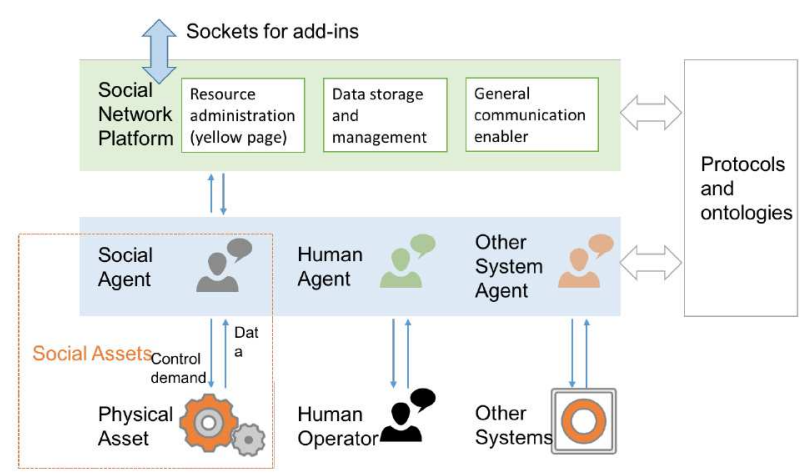

Figure 3. Sketch of the architecture for a Social Network of Collaborating Assets

The rest of the section presents a sketch of the general architecture needed to enable a Social Network of Collaborating Assets, as shown in Fig. 3. The sketch gives the relationships between the building blocks shown in Section 4 and how they are operating. The Social Assets here are composed of: (1) physical assets that directly interact with the environment, perform production tasks, and generate condition data; (2) social agents that act as representatives of physical assets in the cyber world and possess the characteristics described in Section 3. The interface with humans and connection to other systems can be realised through placing agents representing humans or other systems in the Social Network Platform. One way of enabling decision-making support, as add-ins, is illustrated here as an example. Here, the Social Network Platforms provide sockets to allow installation of add-ins with different decision-support functions such as plotting higher-dimensional data analytics.

\section{Application examples}

\section{Collaborative industrial assets for workload sharing}

Directly following the two enhancing characteristics of Social Assets - contextual awareness and cooperation initiative, here we present an example of applications of collaborating industrial assets aiming at improving systemlevel performance by sharing the workload assigned to each other. The two main motivations for this application are:

(1) Parallel machines capable of performing the same type of task are commonly seen in manufacturing plants, enabling the shifting of workload from one machine to the other while still fulfilling the production demand;

(2) The degradation rate of an asset as it operates is closely related by the type and amount of tasks assigned to it (Celen and Djurdjanovic 2016; Hao et al. 2017), making it possible to actively control the residual life of an asset by dynamically changing its workload. An instance of the proposed architecture in the previous section is presented in Fig. 4 that serves the purpose of workload sharing. The functions of the two types of Agents, the Coordinator Agent and Machine Agents, and the Social Network Platform are described as follows:

Social Network Platform - The Social Network Platform in this case has dual roles: a blackboard and a yellow page. As

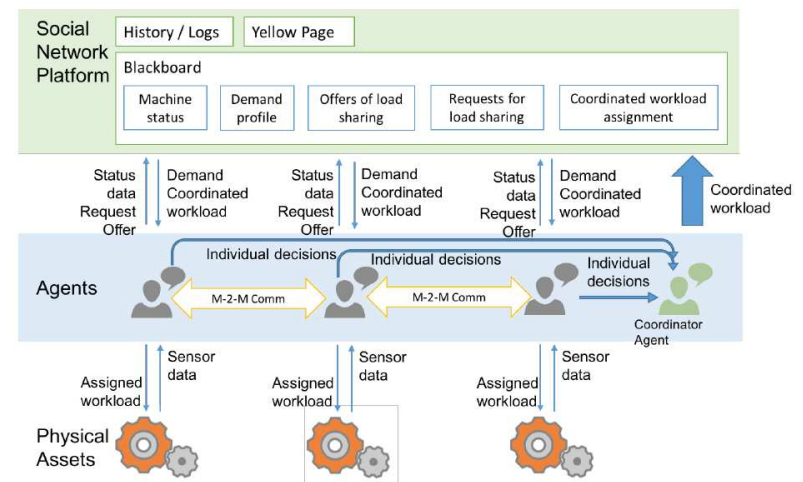

Figure 4. Instance of the Social Networks for Collaborating Assets architecture for workload sharing

a blackboard, it displays the up-to-date status of production resources participating in the network, any request for help posted by close-to-failure machines, and production-related information such as the demand profile in the near future. As a yellow page, it maintains a list of agents registered in the Social Network and keeps logs of their activities if necessary. Coordinator Agent - For computational efficiency, the complex optimisation is performed by machines themselves whereas the Coordinator Agent mainly resolves conflicts of Machine Agents decisions following simple rules or through simple computation.

Machine Agents - Each physical machine has an abstracted representative, a Machine Agent in the virtual world. By checking its own status and the demand profile, the Machine Agents make decisions on the amount of workload to take on, and on the optimal preventive maintenance threshold for itself. The workload decided by Machine Agents individually together with other necessary information needed for coordination is sent to the Coordinator Agent for finalisation. Two other important behaviours of Machine Agents are: seeking help from other machines and offering help to other machines.

For illustration purposes, the flow of data exchange and actions in the scenario where a machine seeks help for workload sharing is presented in Fig. 5

\section{Distributed collaborative prognostics for industrial assets}

As discussed previously in this paper, a Social Network of Collaborating Industrial Assets enables assets to take independent decisions in order to optimise performance and monitoring. A common application enabled by continuous monitoring is failure prognostics, which consists of accurately predicting the time to failure of industrial assets (Goyal and Pabla 2015). The analysis of estimated probabilities for the asset's time to failure allows asset managers to implement optimised maintenance policies which leverage the risk of failure with the costs of preventive and corrective maintenance, and the associated loss of operational time (Dabney et al. 2008). Currently, the optimisation of maintenance policies for large asset fleets is usually done within a centralized architecture, with exceptions such as the Watchdog Agent Djurdjanovic et al. (2003), which implements a hybrid approach using 


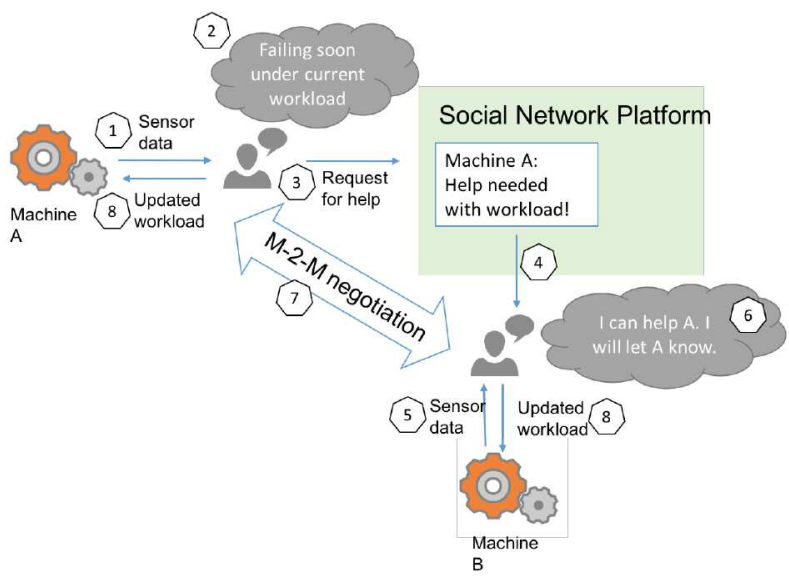

Figure 5. Data and action flow of a machine seeking help for workload sharing

human agents and software agents. A social network of collaborating industrial assets enables the implementation of a new kind of architecture designed for improving prognostics in large asset fleets: distributed collaborative learning, which is aimed at reducing human input as much as possible.

Machine Agents: in this architecture, assets are able to increase their prediction accuracy by using the knowledge gathered by other similar assets in the asset fleet. Each industrial asset has an agent installed that learns separately from the other assets in the network, and collaborates with them sharing relevant data (see Fig. 6). In practice, each asset must be able to continuously compute the following parameters and vectors:

1. A list of inter-asset similarity metrics within the asset fleet. A similarity metric is a positive real number that defines the estimated difference between two assets, and that is assumed to be directly related to the statistical soundness of sharing their data points. This is represented by $d_{i j}$ where $i$ is the asset that will receive the data and $j$ is the asset that will send it. The $N$ assets with smaller metric values will be connected to this asset, the set of assets and interasset connections is defined as an Asset Network (Salvador Palau et al. 2017).

2. Failure diagnostics, performed by evaluation of the sensor data obtained from the Smart Asset.

3. An estimate of its own time to failure with an associated error, both performed using a combination of the asset's own historical data and data from other collaborating assets.

4. An optimisation of its own maintenance policy based on the estimated time to failure considering the constraints given by the Social Network Platform.

Social Network Platform: inter-asset communications and general parameter settings such as the number of collaborating assets, $N$, and constrains in the maintenance policy are set by the Social Network Platform (see Fig. 6). This means that each list of $N$ collaborating assets will be sent and stored in the Social Network Platform in real time.

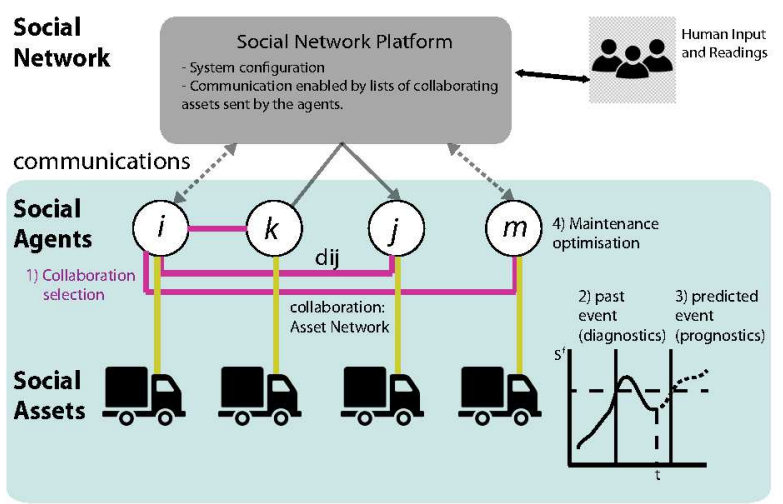

Figure 6. A schematic of the architecture used for distributed collaborative learning and the functions that each agent must be able to perform. In this case, only Social and Human agents and Social assets are needed, as the coordination is performed in a distributed way. Each Social Asset hosts a Social Agent labeled by a letter marked in italics, and each agent calculates its asset similarity metric by means of the parameter $d_{i j}$ (in purple, shown for the asset $i$ ). Each agent must compute four tasks that are described in Section 5.2 and illustrated in the figure. Communications are performed through the social network platform. Here, discontinuous lines indicate communication between the agents and the platform, while the continuous line indicate inter-asset communication enabled by the platform.

This corresponds, as commented in Section. 6.1 to a yellow page function.

This architecture combines the benefits of distributed decision making with the importance of proper sampling in predictive statistics. Preliminary studies have shown that distributed collaborative learning has a positive impact in the maintenance cost function of asset fleets as long as parameters such as the number of collaborating industrial assets, system noise and inter-asset metrics are well defined (Salvador Palau et al. 2017). This approach has applications in large industrial asset fleets with extensive continuous monitoring and local processing power such as gas and wind turbines, automobiles, airplanes and ships.

\section{Guidelines for future work}

Future work should focus on implementation and validation of the proposed framework. Real-world implementation of the application examples is likely to lead to a set of practical problems that are not presented in detail in the framework. In this section, we review some of these practical problems and provide guidelines to overcome them.

1. Lack of asset data: in many industrial systems, sensing is only partially incorporated, and asset characteristics and states are largely unknown. Before considering an implementation of the proposed framework, a review of the asset fleet must be undertaken. Within the fleet, the asset typologies incorporating a condition monitoring system, are readily adaptable to the proposed architecture by either assigning to them a cloud-based agent or endowing them with processing power. Management must then consider which additional asset typologies in the fleet are candidate for the implementation of a condition 
maintenance system. This typically corresponds to complex assets with high maintenance costs.

2. Real-time diagnostics and prognostics: the transition to real-time health management is not without problems. A posteriori diagnostic techniques are often difficult to adapt dynamically, as the dynamic nature of asset condition is usually not considered. A good enabler of cluster-based dynamic diagnostics is the method presented by Dr. Edzel R. Lapira in his PhD thesis (Lapira 2012). Regarding prognostics, the main challenge is including censored data in the algorithms and deciding on the frequency of re-training and prediction. An example of how to overcome these issues can be found in (Martinsson 2016).

3. Appropriate cost metrics: determining repairing or replacement cost in real-time health management systems is not a trivial problem. If the particular industry is used to rigid maintenance scheduling, the proposed approach may seem operationally impossible. It is important to weight the particularities of the industry and asset type in question to decide on whether a real-time approach makes sense. If so, maintenance and replacement costs should be assessed in conversation with suppliers and clients, as all actors involved in the maintenance life-chain may have to change in order to adapt to the new framework.

4. Integrated maintenance and production planning: at the asset level, the trade-off between keeping the asset in a satisfactory condition as well as maximizing its profitability must be considered. Resolving this issue requires a systematic approach to exhaust, classify, and quantify the cost and benefit caused by each operation or maintenance action.

5. Workshop-level decision making: at the workshoplevel, since a fleet of assets of different types and configurations are involved in the production process, it would be worth exploring coordination and negotiation strategies to resolve conflicts of interest between assets to improve system-level performance.

\section{Concluding remarks}

In this paper we have presented what we believe that will be the future paradigm of Asset Management: a Social Network of Collaborating Industrial Assets. In such a network, autonomous assets will take independent decisions and collaborate with each other in a distributed way, coordinated by a central platform. Human input has been reduced as much as possible: setting global constraints and target functions. Therefore, the role of the asset manager will not be any more to choose and optimise performance and maintenance policies but instead to set system constrains and monitor statistics obtained by the Social Network Platform from the asset fleet. In our proposed system, the Social Network Platform will perform the tasks of allowing collaboration, communicating constraints and objective functions to the assets and summarizing fleet information. All the other tasks, such as system optimisation, decision making, collaboration and self monitoring will be performed by agents installed in the assets. The proposed system is then a bottom to top approach, where assets will have certain independence in choosing how to satisfy the constraints and requirements of the Asset Manager. This work forms part of the first steps towards empowering industrial assets with highly autonomous agents in order to reduce costs and increase efficiency.

\section{Funding}

This research was supported by SustainOwner (Sustainable Design and Man- agement of Industrial Assets through Total Value and Cost of Ownership), a project sponsored by the EU Framework Programme Horizon 2020, MSCA- RISE-2014: Marie Skodowska-Curie Research and Innovation Sta Exchange (Rise) (grant agreement number 645733 Sustain-owner H2020-MSCARISE- 2014). This research was also supported by Cambridge Commonwealth, European and International Trust and China Scholarship Council.

\section{Acknowledgements}

The authors wish to thank co-workers in DIAL in the Institute for Manufacturing of University of Cambridge.

\section{Author Biographies}

Hao Li obtained her Bachelor of Engineering degree (Structural Engineering) from Tsinghua University in China in 2014. She joined the Distributed Information and Automation Laboratory at the University of Cambridge, U.K. as a Ph.D candidate in 2015. Her research interest is in asset management, reliability engineering, and distributed optimisation.

Adrià Salvador Palau received his B.Sc. degree in Physics from the Autonomous University of Barcelona, in 2014. He received his M.Sc. degree in 2015 from the University of Bergen (Norway), and is currently a Ph.D. Candidate in the Distributed Information and Automation Laboratory at the University of Cambridge, U.K. He has published several papers in Measurement Science, Physics and Stochastics . His research focuses on optimisation and modelling of systems, usually governed by some degree of randomness.

Ajith Kumar Parlikad is a Senior Lecturer in Industrial Systems at the University of Cambridge. Ajith is the head of the Asset Management Group at the Institute for Manufacturing and is the theme leader on asset management and maintenance at the Centre for Smart Infrastructure and Construction (CSIC). His particular focus is examining how asset information can be used to improve asset performance through effective decision-making. Ajith has published over 100 papers in reputed academic journals and conferences. He is a member of The Institution of Engineering and Technology (IET) Technical Professional Network Committee on Asset Management. In addition, he is currently the chair of the IAM Academic and Research Network.

\section{Declaration of conflicting interests}

The authors declare no potential conflicts of interest with respect to research, authorship and/or publication of this article.

\section{References}

\section{References}

Aissani N, Beldjilali B and Trentesaux D (2009) Dynamic scheduling of maintenance tasks in the petroleum industry: A reinforcement approach. Engineering Applications of Artificial Intelligence 22(7): 1089-1103. 
Al-Fuqaha A, Guizani M, Mohammadi M, Aledhari M and Ayyash M (2015) Internet of things: A survey on enabling technologies, protocols, and applications. IEEE Communications Surveys \& Tutorials 17(4): 2347-2376.

Alam KM, Saini MK and El-Saddik A (2015) Toward social internet of vehicles: concept, architecture, and applications. IEEE Access 3: 343-357.

Atzori L, Lera A and Morabito G (2014) From smart objects to social objects: the next evolutionary step of the internet of things. IEEE Communications Magazine 52(1): 97-105.

Atzori L, Lera A, Morabito G and Nitti M (2012) The social internet of things (siot) - when social networks meet the internet of things: concept, architecture and network characterisation. Computer Networks 56(16): 3594-3608.

Bleecker J (2006) A manifesto for networked objects - cohabiting with pigeons, arphids and aibos in the internet of things. In: Proc. of the 13th International Conference on HumanComputer Interaction with Mobile Devices and Services, MobileHCI. pp. 1-17.

Brintrup A, McFarlane D, Ranasinghe D, Sanchez Lopez T and Owens K (2011) Will intelligent assests take off? toward selfserving aircraft. IEEE Intelligent Systems 26(3): 66-75.

Cai H, Xu LD, Xu B, Xie C, Qin S and Jiang L (2014) Iot-based configurable information service platform for product lifecycle management. IEEE Transactions on Industrial Informatics 10(2): 1558-1567.

Celen $M$ and Djurdjanovic D (2016) Integrated maintenance decision-making and product sequencing in flexible manufacturing systems. Journal of Manufacturing Science and Engineering 137(4): 1-15.

Cerrada M, Cardillo J, Aguilar J and Faneite R (2007) Agents-based design for fault management systems in industrial processes. Computers in Industry 58(4): 313-328.

Coudert T, Grabot B and Archimède B (2002) Production/maintenance cooperative scheduling using multi-agents and fuzzy logic. International Journal of Production Research 40(18): 4611-4632.

Dabney T, Hernandez L, Scandura PA and Vodicka R (2008) Enterprise health management framework - a holistic approach for technology planning, $\mathrm{r}$ and d collaboration and transition. In: International Conference on Prognostics and Health Management, 2008. Denver, CO, USA: IEEE.

De Saulles M (2016) The Internet of Things and Business by Martin De Saulles. Routledge, 2016.

Djurdjanovic D, Lee J and Ni J (2003) Watchdog Agent - an infotronics-based prognostics approach for product performance degradation assessment and prediction. Advanced Engineering Informatics 17(3-4): 109-125.

Feng Q, Li S and Sun B (2012) An intelligent fleet condition-based maintenance decision making method based on multi-agent. International Journal of Prognostics and Health Management 3(1): 1-11.

Fortino G, Guerrieri A, Russo W, Bucci VP and Cs R (2012) Agent-oriented smart objects development. In: Proceedings of the 2012 IEEE 16th Internaitonal Conference on Computer Supported Cooperative Work in Design (CSCWD). Wuhan: IEEE, pp. 907-912.

Geerts GL and O'Leary DE (2014) A supply chain of things: The eaglet ontology for highly visible supply chains. Decision Support Systems 63: 3-22.
Gerla M, Lee EK, Pau G and Lee U (2014) Internet of vehicles: From intelligent grid to autonomous cars and vehicular clouds. In: Internet of Things (WF-IoT), 2014 IEEE World Forum on. Seoul, South Korea: IEEE.

Goyal D and Pabla BS (2015) Condition based maintenance of machine tools - a review. CIRP Journal of Manufacturing Science and Technology 10: 24-35.

Gubbi J, Buyya R, Marusic S and Palaniswami M (2013) Internet of things (iot): A vision, architectural elements, and future directions. Future Generation Computer Systems 29(7): 16451660 .

Guo B, Zhang D, Wang Z, Yu Z and Zhou X (2013) Opportunistic iot: Exploring the harmonious interaction between human and the internet of things. Journal of Network and Computer Applications 36(6): 1531 - 1539.

Hao L, Liu K, Gebraeel N and Shi J (2017) Controlling the residual life distribution of parallel unit systems through workload adjustment. IEEE Transactions on Automation Science and Engineering 14(2): 1042-1052.

Iung B, Levrat E, Crespo Marquez A and Erbe H (2009) Conceptual framework for e-maintenance: Illustration by e-maintenance technologies and platforms. Annual Reviews in Control 33(2): 220-229.

Jiang C and Sheng Z (2009) Case-based reinforcement learning for dynamic inventory control in a multi-agent supply-chain system. Expert Systems with Applications 36(3): 6520-6526.

Khelifati SL and Benbouzid-Sitayeb F (2013) A multi-agent scheduling approach for the joint scheduling of jobs and maintenance operations in the flow shop sequencing problem. International Journal of Intelligent Engineering Informatics 2(1): 47-70.

Kortuem G, Kawsar F, Sundramoorthy V and Fitton D (2010) Smart objects as building blocks for the internet of things. IEEE Internet Computing 14(1): 44-51.

Kosmatos EA, Tselikas ND and Boucouvalas AC (2011) Integrating rfids and smart objects into a unified internet of things architecture. Advances in Internet of Things 1(1): 5-12.

Kranz M, Roalter L and Michahelles F (2010) Things that twitter: social networks and the internet of things. In: What can the Internet of Things do for the Citizen (CIoT) Workshop at The Eighth International Conference on Pervasive Computing (Pervasive 2010). Helsinki, Finnland.

Lapira ER (2012) Fault detection in a network of similar machines using clustering approach. $\mathrm{PhD}$ Thesis, University of Cincinnati, Cincinnati, OH, USA.

Lee J, Bagheri B and Kao HA (2015) A cyber-physical systems architecture for industry 4.0-based manufacturing systems. Manufacturing Letters 3: 18-23.

Lemaignan S, Siadat A, Dantan JY and Semenenko A (2006) Mason: A proposal for an ontology of manufacturing domain. Proceedings - DIS 2006: IEEE Workshop on Distributed Intelligent Systems - Collective Intelligence and Its Applications : 195-200.

Li Y, Hou M, Liu H and Liu Y (2012) Towards a theoretical framework of strategic decision, supporting capability and information sharing under the context of internet of things. Information Technology and Management 13(4): 205-216.

Lim MK, Zhang Z and Goh WT (2009) An iterative agent bidding mechanism for responsive manufacturing. Engineering 
Applications of Artificial Intelligence 22(7): 1068-1079. DOI: 10.1016/j.engappai.2008.12.003.

Mangina E, McArthur SDJ and McDonald J (2000) Autonomous agent for distributed problem solving in condition monitoring. Intelligent Problem Solving. Methodologies and Approaches: 13th International Conference on Industrial and Engineering Applications of Artificial Intelligence and Expert Systems : 683-693.

Martinsson E (2016) WTTE-RNN: Weibull time to event recurrent neural network. Master's Thesis, University of Gothenburg, Gothenburg, Sweden.

McArthur SDJ, Booth CD, McDonald JR and McFadyen IT (2005) An agent-based anomaly detection architecture for condition monitoring. IEEE Transactions on Power Systems 20(4): 16751682. DOI:10.1109/TPWRS.2005.857262.

McArthur SDJ, Strachan SM and Jahn G (2004) The design of a multi-agent transformer condition monitoring system. IEEE Transactions on Power Systems 19(4): 1845-1852. DOI:10. 1109/TPWRS.2004.835667.

Ortiz AM, Hussein D, Park S, Han SN and Crespi N (2014) The cluster between internet of things and social networks: Review and research challenges. IEEE Internet of Things Journal 1(3): 206-215.

Ouelhadj D, Hanachi C and Bouzouia B (2000) Multiagent architecture for distributed monitoring in flexible manufacturing systems (FMS). In: Proceedings of the 2000 IEEE International Conference on Robotics and Automation, April. San Francisco, CA. ISBN 0780358864, pp. 2416-2421.

Salvador Palau A, Liang Z, Lütgehetmann D and Parlikad AK (2017) Collaborative prognostics in social asset networks. Submitted to Future Generation Computer Systems.

Sánchez López T, Ranasinghe DC, Harrison M and McFarlane D (2012) Adding sense to the Internet of Things: an architecture framework for Smart Object systems. Personal and Ubiquitous Computing 16(3): 291-308.

Schurgot MR, Comaniciu C and Jaffr K (2012) Beyond traditional DTN routing : social networks for opportunistic communication. IEEE Communications Magazine 50(7): 155162.

Tuyls K and Weiss G (2012) Multiagent learning: Basics, challenges, and prospects. AI Magazine 33(3): 41-52.

Ungurean I, Gaitan NC and Gaitan VG (2014) An iot architecture for things from industrial environment. In: Соттиnications(COMM), 2014 10th International Conference on. Bucharest, Romania: IEEE, pp. 1-4.

Van Kranenburg R (2008) The Internet of Things: a critique of ambient technology and the all-seeing network of RFID, volume 2. Institute of Network Cultures. ISBN 9789078146063.

Zaslavsky A, Perera C and Georgakopoulos D (2013) Sensing as a service and big data. In: Proceedings of the International Conference on Advances in Cloud Computing (ACC), Bangalore, India, July, 2012.

Zhang Y, Wang W, Liu S and Xie G (2014) Real-time shop-floor production performance analysis method for the internet of manufacturing things. Advances in Mechanical Engineering 6: $1-10$.

Zhou R, Fox B, Lee HP and Nee AYC (2004) Bus maintenance scheduling using multi-agent systems. Engineering Applications of Artificial Intelligence 17(6): 623-630. 